University of Wollongong

Research Online

Faculty of Engineering and Information

Faculty of Engineering and Information

Sciences - Papers: Part B

Sciences

2016

Characterization of angular detection dependence of prompt gamma-rays with respect to the Bragg peak in a water phantom using proton beam irradiations

Melek Zarifi

University of Wollongong, mz659@uowmail.edu.au

Susanna Guatelli

University of Wollongong, susanna@uow.edu.au

David Bolst

University of Wollongong, dbolst@uow.edu.au

Brian F. Hutton

University of Wollongong, b.hutton@ucl.ac.uk

Anatoly B. Rosenfeld

University of Wollongong, anatoly@uow.edu.au

See next page for additional authors

Follow this and additional works at: https://ro.uow.edu.au/eispapers1

Part of the Engineering Commons, and the Science and Technology Studies Commons

Research Online is the open access institutional repository for the University of Wollongong. For further information contact the UOW Library: research-pubs@uow.edu.au 


\title{
Characterization of angular detection dependence of prompt gamma-rays with respect to the Bragg peak in a water phantom using proton beam irradiations
}

\begin{abstract}
The rationale for utilizing the prompt gamma (PG) signal for in vivo proton beam range verification is such that the PG fall-off distribution along the beam path is associated with the dose profile in the Bragg peak (BP) distal fall-off region. Quantitative characterization of this association, particularly with respect to the $\mathrm{BP}$, is of great importance to assess its limitation and aid in the development of a clinically reliable PG imaging system to maximize PG detection. In this work we investigate the angular dependence of PG detection with respect to the BP for in vivo beam range verification in proton radiation therapy. Geant4 Monte Carlo simulations have been used to study the energy spectral and spatial characteristics of the PG signal from high-energy proton beam irradiations. A cylindrical water phantom $(\varphi 30 \mathrm{~cm} \times 50 \mathrm{~cm})$ with an ideal detecting cylinder $(\varphi 100 \mathrm{~cm} \times 50 \mathrm{~cm})$ coaxially surrounding the phantom has been used in the simulation. The angular dependence of PG detection as a function of beam energy and PG energy has been characterized with respect to the BP. Our results show that there exists an angular preference for PG detection, which has a strong dependence on the beam energy. As the beam energy increases, the longitudinal angular preference for PG detection becomes increasingly backward with respect to the BP position. This implies that the detector with sufficient longitudinal angular coverage is desired for the BP tracking, especially for the Spread-Out Bragg Peak tracking.

\section{Disciplines}

Engineering | Science and Technology Studies

\section{Publication Details}

Zarifi, M., Guatelli, S., Bolst, D., Hutton, B., Rosenfeld, A. \& Qi, Y. (2016). Characterization of angular detection dependence of prompt gamma-rays with respect to the Bragg peak in a water phantom using proton beam irradiations. 2016 IEEE Nuclear Science Symposium, Medical Imaging Conference and Room-Temperature Semiconductor Detector Workshop (NSS/MIC/RTSD) (pp. 582-586). United States: IEEE.
\end{abstract}

\section{Authors}

Melek Zarifi, Susanna Guatelli, David Bolst, Brian F. Hutton, Anatoly B. Rosenfeld, and Yujin Qi 


\title{
Characterization of Angular Detection Dependence of Prompt Gamma-rays with Respect to the Bragg Peak in a Water Phantom Using Proton Beam Irradiations
}

\author{
M. Zarifi, S. Guatelli, D. Bolst, B. Hutton, A. Rosenfeld, Y. Qi
}

\begin{abstract}
The rationale for utilizing the prompt gamma (PG) signal for in vivo proton beam range verification is such that the PG fall-off distribution along the beam path is associated with the dose profile in the Bragg peak (BP) distal fall-off region. Quantitative characterization of this association, particularly with respect to the $\mathrm{BP}$, is of great importance to assess its limitation and aid in the development of a clinically reliable PG imaging system to maximize PG detection. In this work we investigate the angular dependence of $P G$ detection with respect to the $\mathrm{BP}$ for in vivo beam range verification in proton radiation therapy. Geant4 Monte Carlo simulations have been used to study the energy spectral and spatial characteristics of the PG signal from high-energy proton beam irradiations. A cylindrical water phantom $(\phi 30 \mathrm{~cm} \times 50 \mathrm{~cm})$ with an ideal detecting cylinder $(\phi 100 \mathrm{~cm} \times 50 \mathrm{~cm})$ coaxially surrounding the phantom has been used in the simulation. The angular dependence of PG detection as a function of beam energy and PG energy has been characterized with respect to the BP. Our results show that there exists an angular preference for PG detection, which has a strong dependence on the beam energy. As the beam energy increases, the longitudinal angular preference for PG detection becomes increasingly backward with respect to the $B P$ position. Although the maximum of the PG measurement associated with the $B P$ is difficult, it can be optimized with energy and angular detection preferences.
\end{abstract}

\section{INTRODUCTION}

$\mathrm{P}$ rompt gamma (PG) rays, as a secondary by-product generated inside the patient from proton beam irradiations, have been proposed for in vivo beam range verification to monitor the dose delivery during the treatments [1]-[3]. The PG imaging method has a unique advantage to overcome the limitation of the in vivo positron emission tomography (PET) method because it can enable real-time tracking of the Bragg peak (BP) during beam delivery with no washout effect [4]. Significant research efforts have been made in the development of clinically suitable and reliable PG imaging technology for in vivo beam range verification [5]-[10].

Manuscript received December 10, 2016. This work was supported in part by the URC small grant of University of Wollongong.

M. Zarifi is with the Centre for Medical Radiation Physics, University of Wollongong, NSW, Australia, (e-mail: mz659@uowmail.edu.au).

S. Guatelli is with the Centre for Medical Radiation Physics, University of Wollongong, NSW, Australia, (e-mail: susanna@uow.edu.au).

D. Bolst is with the Centre for Medical Radiation Physics, University of Wollongong, NSW, Australia, (e-mail: db001@uowmail.edu.au).

B. Hutton is with the Institute of Nuclear Medicine, University of College London, London, UK, (e-mail: b.hutton@ucl.ac.uk).

A. Rosenfeld is with the Centre for Medical Radiation Physics, University of Wollongong, NSW, Australia, (e-mail: anatoly@uow.edu.au).

Y. Qi is with the Centre for Medical Radiation Physics, University of Wollongong, NSW, Australia, (e-mail: yujin@uow.edu.au).
The rationale for utilizing PG signal for in vivo beam range verification is based on the fact that the PG fall-off distribution along the beam path is associated with the dose profile in the BP distal fall-off region [1], [2]. However, their relationship has complicated characteristics because they result from different physical processes. The dose deposition is mainly the result of the Coulomb interaction between the proton beam and tissue's electrons while the PG emissions result from nuclear interactions between the proton beam and tissue's nuclei. There is no exact one-on-one relationship existing between PG emission and the dose deposition. As pointed out in [11] there are many factors influencing the accuracy of beam range estimation using PG emission. Quantitative characterization of the correlation between these factors, particularly with respect to the $\mathrm{BP}$, is of great importance to assess its limitation and aids in the design of a clinically reliable PG imaging system.

The energy spectral characteristics of PG emissions from proton irradiations have been extensively studied and verified by several research groups [12], [13]. Proton-induced PG emission exhibits a broad energy spectrum in a range of 2-15 $\mathrm{MeV}$ and is dominated by a number of characteristic gammalines that depend on the elements of the irradiated tissue composite [14]. A recent study from Verburg et al. [15] shows that identifying those discrete PG lines could provide several benefits to improve the accuracy and efficiency of beam range verification. Quantitative measurements of the characteristic PG rays can be used for precise proton range verification in the presence of tissue with an unknown composition.

Our previous simulation study [16], [17] has characterized the relationship between the PG emission fall-off and the BP distal fall-off in water and PMMA phantoms. Strong correlation with high accuracy between the characteristic PG emissions and the BP has been observed. Submillimeter shift of the BP position has been identified from the emitted PG distribution, but the detectable PG distributions out of the phantoms are significantly different from the emission distributions. How the detectable PG yields out of the patient affect the BP tracking needs to be assessed. Further investigation of the spatial characteristics of the detectable PG distribution could provide valuable information to optimize the PG measurement associated with the BP tracking.

In this work, we investigate the angular distribution of the detectable PG yields out of a water phantom with respect to the BP to maximize the PG detection efficiency for real-time beam range verification in proton beam irradiations. Extensive Geant4 Monte Carlo simulations have been used to study the angular dependence of the PG measurement with respect to 
the $\mathrm{BP}$ as a function of proton beam energy and PG energy window.

\section{METHODOLOGY}

Geant4 [18], [19], version 10.00 was adopted to characterize the PG emission from proton pencil beam irradiations of a homogeneous water phantom $\left(\mathrm{H}_{2} \mathrm{O}\right.$, density of $1 \mathrm{~g} / \mathrm{cm}^{3}$ ). The cylindrical phantom has a radius of $30 \mathrm{~cm}$ and height $50 \mathrm{~cm}$. The geometrical setup of the simulations is shown in Fig. 1. The proton pencil beam is incident normally on the surface of the phantom, along the cylinder axis (z-axis). Monoenergetic proton beams of energy 100, 120, 140, 160, 180 and $200 \mathrm{MeV}$ were simulated.

The Geant4 physicslist includes both electromagnetic (Livermore Low Energy Package) and hadronic physics (QGSP_BIC_HP for protons, neutrons and pions, Binary Ion Cascade model for ions). The production threshold of secondary particles was fixed to $0.05 \mathrm{~mm}$.

As shown in Fig. 1, a dummy cylinder (called detection cylinder) with radius $50 \mathrm{~cm}$ is modelled coaxially surrounding the phantom and registers secondary radiation from the proton-induced phantom.

The output of the simulation consists of the energy and location of the secondary photons and neutrons when they originate within the phantom and when reaching the detection cylinder's surface. The preferential position for PG measurement, using the detection cylinder, is denoted with the angle $\theta_{\text {pref. }}$ This angle $\theta_{\text {pref }}$ is formed between $\mathrm{P}$, the most probable point on the detection cylinder traversed by the photons and the normal to the main axis of the phantom passing by the proton beam range, see Fig. 1. $\theta$ represents all other angles subtended from the normal.

The main characteristics of PG emission and detection in terms of the beam energy and PG energy window dependence can be investigated.
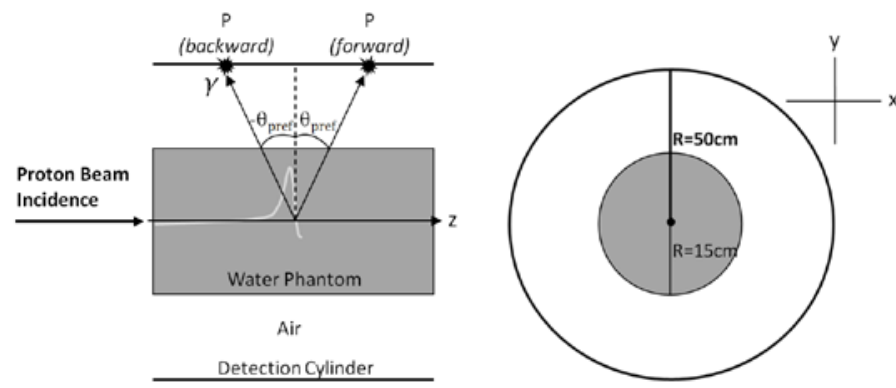

Fig. 1. Geometrical setup of the Geant4 simulation, cross-section view (left) and side-view (right). The proton pencil beam is incident normally on the surface of the phantom, along the cylinder axis (z-axis). The detection cylinder is also shown, with the preferential position of PG detection determined with the angle $\theta_{\text {pref }}$.

\section{RESULTS}

\section{A. PG Emission Characteristics in the Phantom}

The calculated beam ranges at the 50\% BP distal fall-off for the proton pencil beams of 100,120,140, 160, 180 and 200 $\mathrm{MeV}$ in the water phantom are approximately 76, 106, 139, 176, 215 and $258 \mathrm{~mm}$, respectively.
Fig. 2 shows the energy spectra of gamma photons produced in the water phantom for proton beam energies from 100-200 MeV. These energy spectra show consistent characteristics over a wide beam energy range and have several distinguishable emission lines produced by non-elastic proton-nuclei interactions with major constituent elements of the phantom material. These characteristic spectral lines include a positron annihilation gamma peak at $0.511 \mathrm{MeV}$ $\left({ }^{15} \mathrm{O}\right)$, a $2.22 \mathrm{MeV}$ gamma peak from the capture of secondary thermal neutrons by Hydrogen, and three prominent PG lines of 4.44, 5.21 and 6.13 MeV. The 4.44 MeV PG emission line is the most prominent in both phantoms as it originates from the de-excitations of ${ }^{12} \mathrm{C}^{*}$, arising from proton nuclear interactions with oxygen nuclei. The 5.21 MeV PG emission line is from ${ }^{15} \mathrm{O}^{*}$ de-excitations, and the $6.13 \mathrm{MeV}$ PG line is from ${ }^{16} \mathrm{O}^{*}$ de-excitations. Then the characteristics of the individual PG lines are further investigated by employing three equal-width energy windows of 4.2-4.6 MeV, 5.0-5.4 $\mathrm{MeV}$, and 5.9-6.3 MeV. An additional larger energy window of 4.2-6.3 MeV is also used to look into the overall effect from all three PG lines. The angular PG yield distribution and correlation with the BP in each energy window are quantified.

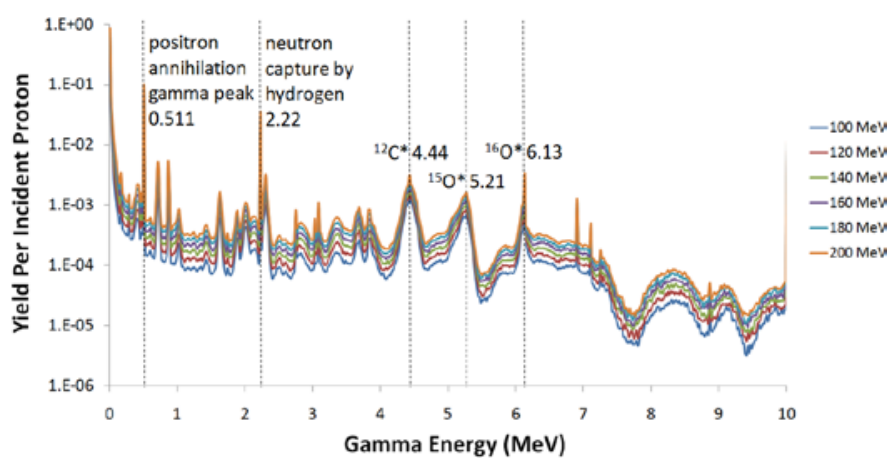

Fig. 2. Monte Carlo calculated energy spectra of gamma photons generated in the water phantom from different proton beam energies of 100-200 MeV.

\section{B. Preferential Angular Dependence for PG Measurement}

By virtue of an ideal detection cylinder coaxially surrounding the phantom, the angular distribution of the detectable PG yields has been characterized. As expected, the detectable PG distributions show an isotropically azimuthal distribution, but non-isotropically longitudinal distribution. Figs. 3, 4 and 5 show typical non-isotropically longitudinal angular distributions of the PG yields out of the water phantom with different PG energy windows of 4.2-4.6, 5.05.4, 5.9-6.3 and 4.2-6.3 MeV respectively, at different proton beam energies of 100, 160 and $200 \mathrm{MeV}$. Unlike the emitted longitudinal distribution within the phantom [16], [17], there is no sharp fall-off of the detectable PG yield distribution in the BP distal fall-off region. However it shows that there exists an angular preference for PG measurement to maximize the geometrical efficiency of PG detection associated with the BP. The preferential angle at the maximum PG yield position, $\theta_{\text {pref}}$, is determined by a curve fitting with a $4^{\text {th }}$-order polynomial function. 

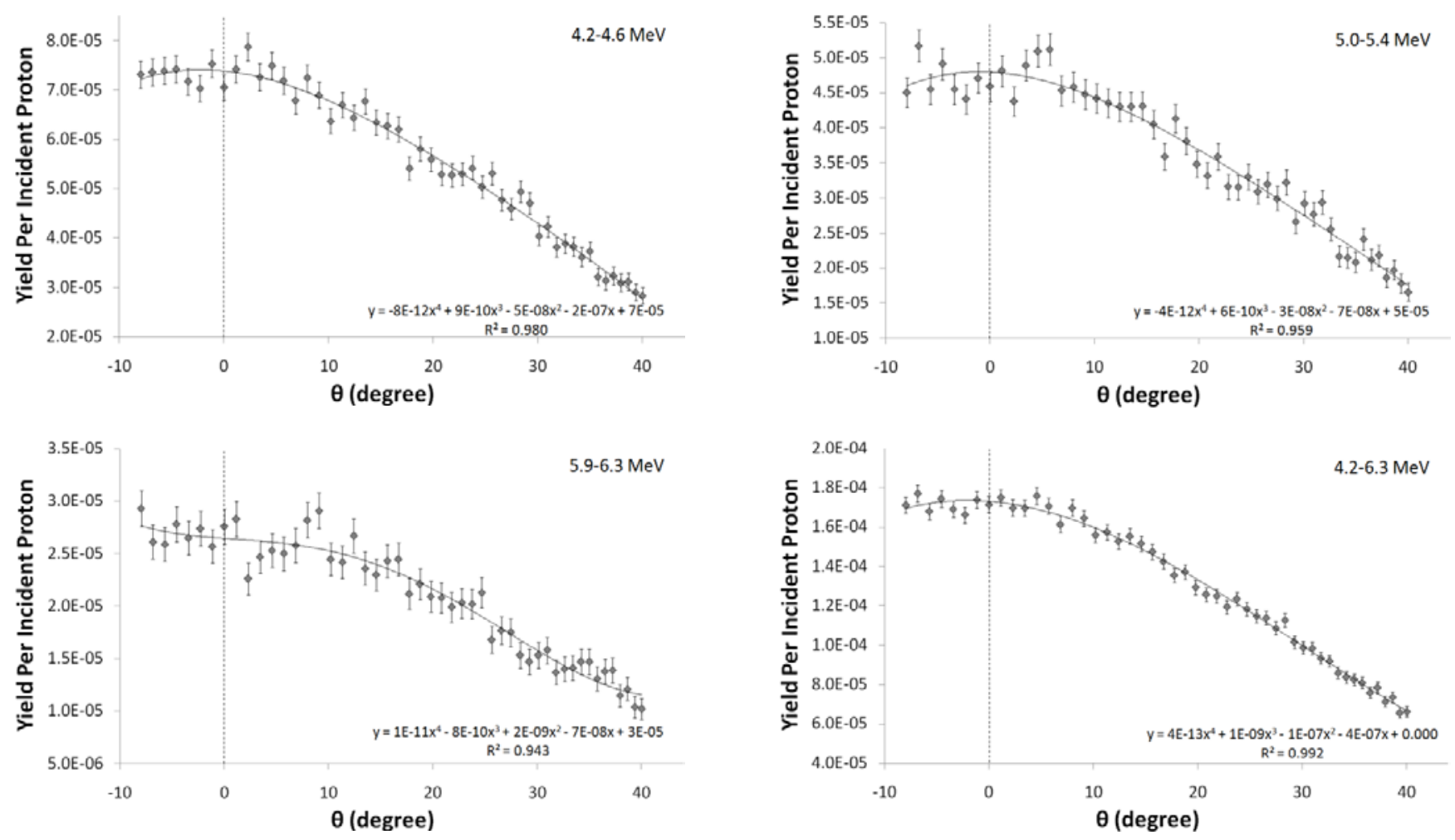

Fig. 3. The longitudinal angular distributions of the PG yields from 4.2-4.6, 5.0-5.4, 5.9-6.3 and 4.2-6.3 MeV PG energy windows reaching the detection cylinder at the proton beam energy of $100 \mathrm{MeV}$. The dash-line denotes the $50 \% \mathrm{BP}$ fall-off position. The solid-line curves are the curve fitting with a $4^{\text {th }}$-order polynomial function.
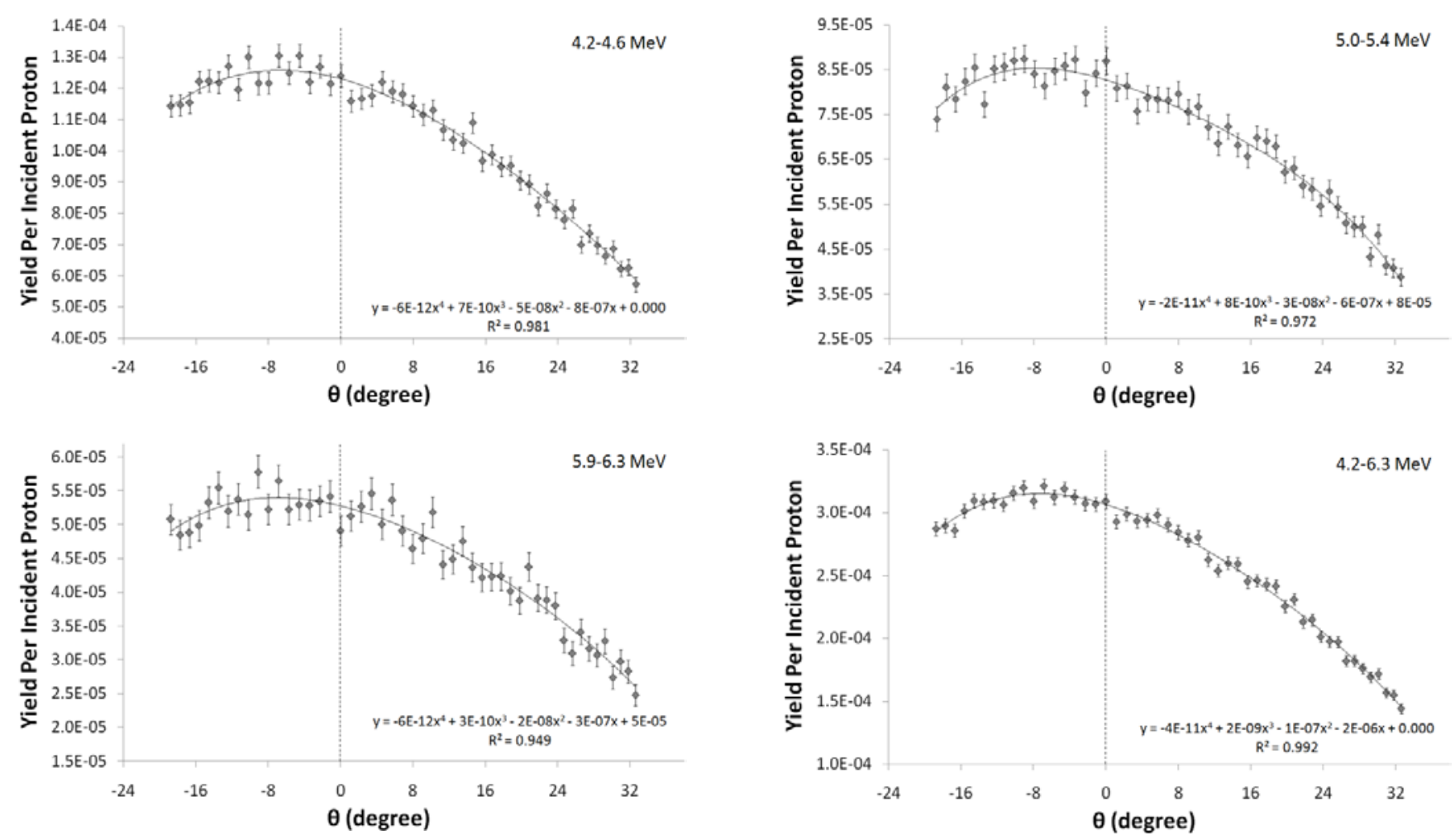

Fig. 4. The longitudinal angular distributions of the PG yields from 4.2-4.6, 5.0-5.4, 5.9-6.3 and 4.2-6.3 MeV PG energy windows reaching the detection cylinder at the proton beam energy of $160 \mathrm{MeV}$. The dash-line denotes the $50 \% \mathrm{BP}$ fall-off position. The solid-line curves are the curve fitting with a $4^{\text {th }}$-order polynomial function. 

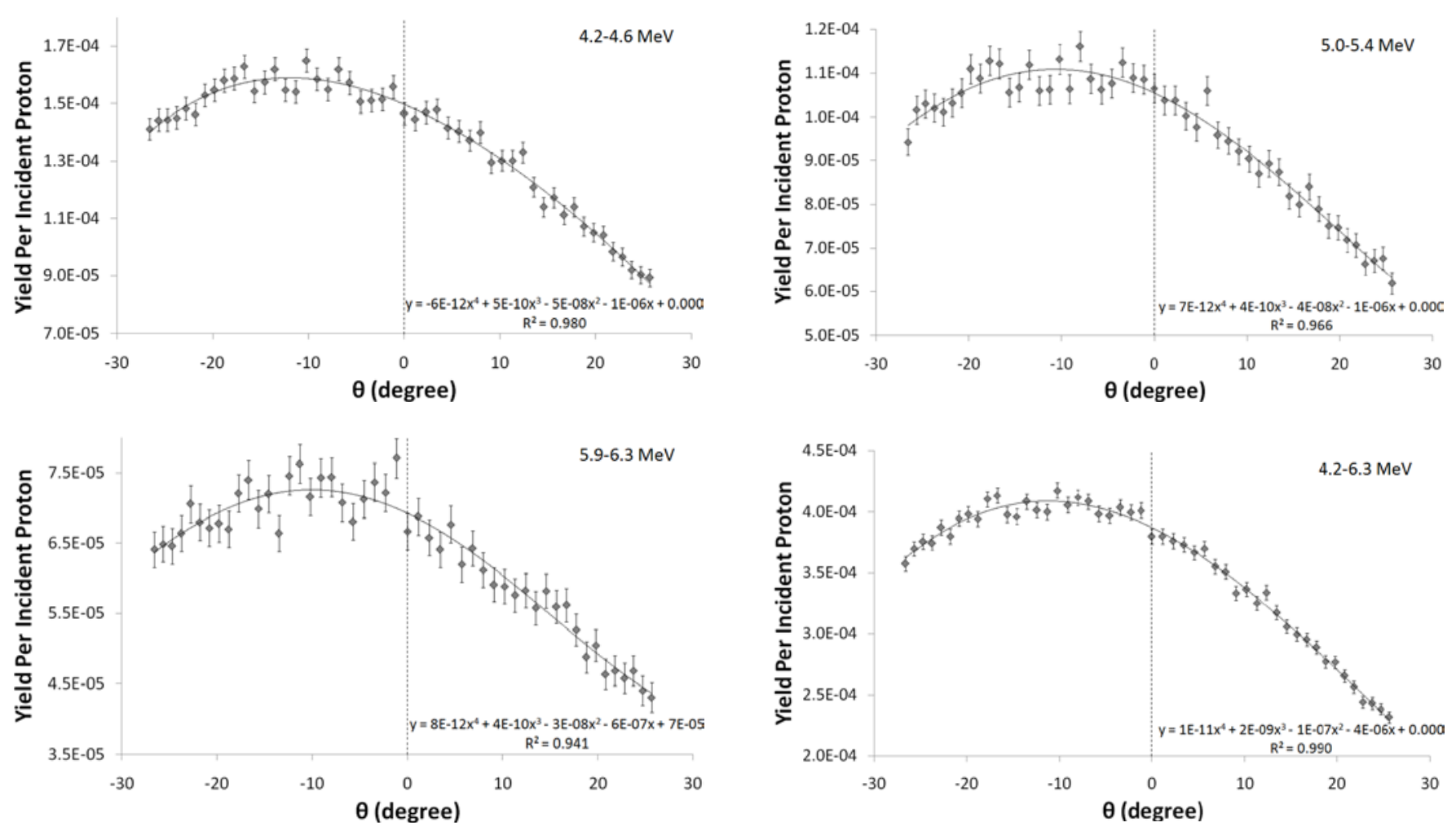

Fig. 5. The longitudinal angular distributions of the PG yields from 4.2-4.6, 5.0-5.4, 5.9-6.3 and 4.2-6.3 MeV PG energy windows reaching the detection cylinder at the proton beam energy of $200 \mathrm{MeV}$. The dash-line denotes the $50 \% \mathrm{BP}$ fall-off position. The solid-line curves are the curve fitting with a $4^{\text {th }}$-order polynomial function.

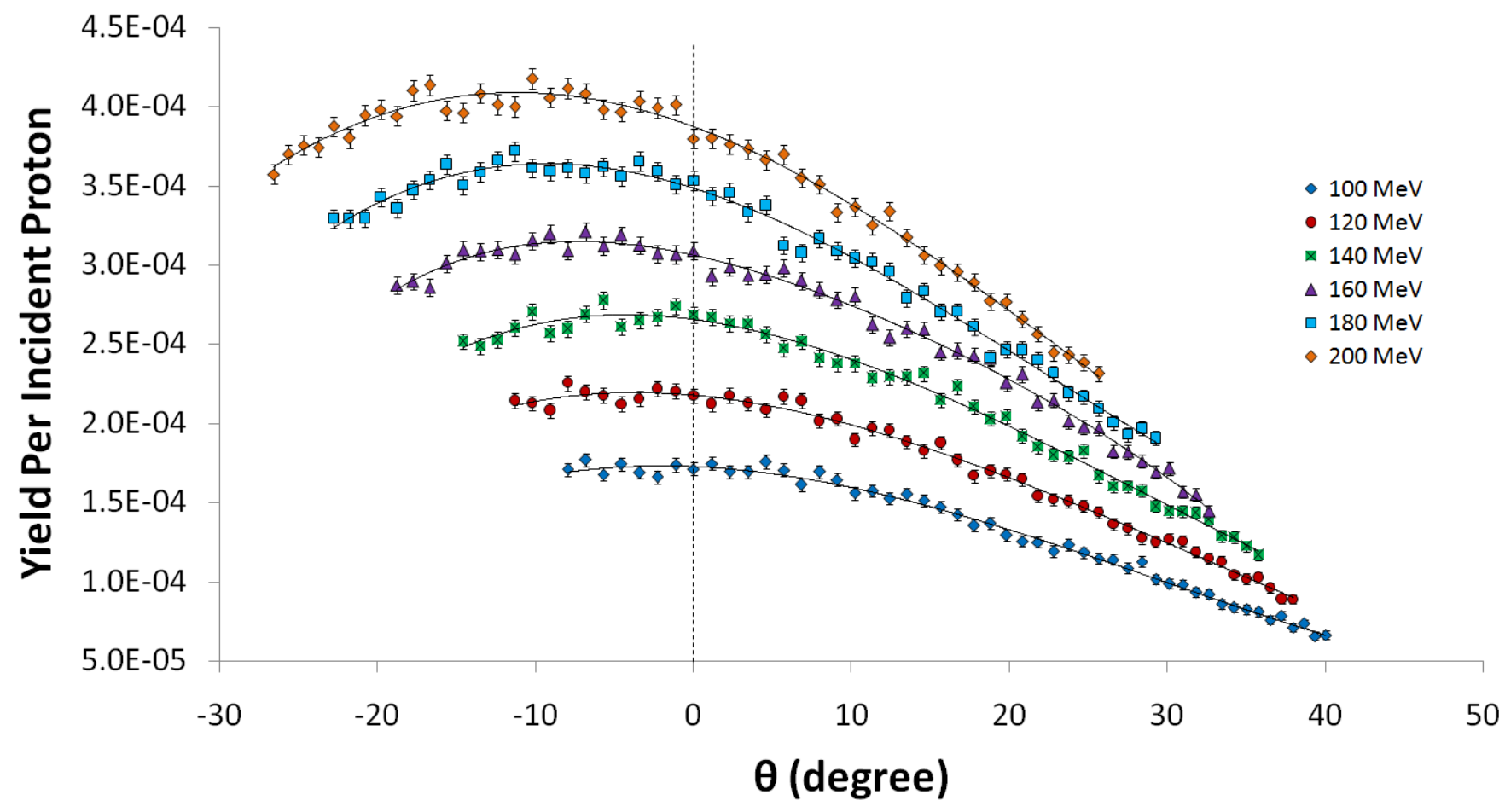

Fig. 6. The PG yield in the 4.2-6.3 MeV PG energy window reaching the detection cylinder as a function of the angle $\theta$ with respect to the proton beam range in the phantom at the $50 \%$ BP fall-off position (denoted by the dash-line) at different proton beam energies of 100,120 , 140, 160, 180 and $200 \mathrm{MeV}$. The bin width for yield values is $\sim 1^{\circ}$. 
Fig. 6 shows the dependence of the longitudinal angular distribution of the detectable PG yields on the proton beam energies. As expected, the angular distributions of PG yields show strong dependence on the proton beam energy. As the beam energy increases, the longitudinal angular distributions are increasingly backward peaked with respect to the BP positions. Table I shows the extracted angular preferences for PG measurements in different PG energy windows as a function of the proton beam energy.

TABLE I. LONGITUDINAL ANGULAR PREFERENCE OF TOTAL GAMMA AND PG EMISSIONS WITH RESPECT TO THE BP FOR EACH PROTON BEAM ENERGY.

\begin{tabular}{|c|c|c|c|c|c|}
\hline \multirow{2}{*}{$\begin{array}{c}\text { Proton } \\
\text { beam } \\
\text { energy } \\
(\mathrm{MeV})\end{array}$} & \multicolumn{5}{|c|}{$\begin{array}{c}\text { Angular preferences, } \theta_{\text {pref }}\left( \pm 1^{\circ}\right) \text {, in different gamma } \\
\text { photon energy windows }(\mathrm{MeV})\end{array}$} \\
\cline { 2 - 6 } & No & $4.2-4.6$ & $5.0-5.4$ & $5.9-6.3$ & $4.2-6.3$ \\
\hline 100 & 1 & -2 & -1 & 0 & -2 \\
\hline 120 & 0 & -5 & -2 & -2 & -3 \\
\hline 140 & -3 & -6 & -3 & -3 & -5 \\
\hline 160 & -6 & -7 & -8 & -7 & -8 \\
\hline 180 & -8 & -9 & -8 & -9 & -10 \\
\hline 200 & -10 & -12 & -11 & -10 & -11 \\
\hline
\end{tabular}

*Negative values represent backward peaked emission while positive values represent forward peaked emission.

The longitudinal angular preference data show a strong dependence on the proton beam energy. At the low proton beam energy of $100 \mathrm{MeV}$, the angular preference $\left(\theta_{\text {pref }}\right)$ for 4.2-6.3 MeV gamma photons is around $-2^{\circ}$, which is slightly backward with respect to the BP position. As the beam energy increases, the angular preference to maximize the number of detectable PG rays gets increasingly backward with respect to their BP position. At the high beam energy of $200 \mathrm{MeV}$, the angular preference $\left(\theta_{\text {pref }}\right)$ for 4.2-6.3 MeV gamma photons becomes around $-11^{\circ}$, which is significantly backward with respect to the $\mathrm{BP}$ position.

The angular preference for PG measurements also shows considerable variations with PG energy windows. The data show that selecting specific PG energy windows $\theta_{\text {pref }}$ changes within 2-3 degrees.

\section{DISCUSSION AND CONCLUSION}

By virtue of an ideal detection cylinder, the angular characteristics for PG measurement from a water phantom using $100-200 \mathrm{MeV}$ proton beam irradiations have been investigated in this study. The non-isotropic longitudinal distribution of PG emission results in an angular preference for PG detection in the detection cylinder, which could be utilized for maximizing the geometrical efficiency of PG measurement. Our results show that there is no sharp fall-off of the detectable PG yield distribution corresponding to the position of the proton beam range.

However, there exists an angular preference for PG measurement with respect to the BP position, which has a strong dependence on the proton beam energy. As the beam energy increases, the angular preference to maximize the number of detectable PG rays becomes increasingly backward peaked with respect to their BP position. This implies that the detector with sufficient longitudinal angular coverage is needed for the BP tracking, especially for the Spread-Out Bragg Peak tracking.

Although it seems impossible to maximize the PG detection associated with the BP position, the PG measurement can be optimized with energy and angular detection preferences. The design of a PG detector with particular energy and geometrical acceptance preferences is desirable. Further work is necessary if the detection of PG associated with the $\mathrm{BP}$ is to be optimized with a specific PG detector system.

\section{REFERENCES}

[1] C. H. Min, C. H. Kim, M. Y. Youn, J. W. Kim, "Prompt gamma measurements for locating the dose falloff region in the proton therapy," Appl. Phys. Lett., vol. 89, no. 18, pp. 183517, 2006.

[2] M. Testa et al., "Real-time monitoring of Bragg-peak position in ion therapy by means of single photon detection, "Radiat. Environ. Biophys., vol. 49, no. 3, pp. 337-343, 2010.

[3] A.C. Knopf, A. Lomax, "In vivo proton range verification: a review," Phys. Med. Biol., vol. 58, no. 15, pp. 131-160, 2013.

[4] M. Moteabbed, S. Espana, H. Paganetti, "Monte Carlo patient study on the comparison of prompt gamma and PET imaging for range verification in proton therapy," Phys. Med. Biol., vol. 56, no. 4, pp. 1063-1082, 2011.

[5] J. Smeets et al., "Prompt gamma imaging with slit camera for real-time range control in proton therapy," Phys. Med. Biol., vol. 57, no. 11, pp. 3371-405, 2012.

[6] V. Bom, L. Joulaeizadeh, F. Beekman, "Real-time prompt gamma monitoring in spot-scanning proton therapy using imaging through a knife-edge-shaped slit," Phys. Med. Biol., vol. 57, no. 2, pp. 297-308, 2012.

[7] D. Mackin, S. Peterson, S. Beddar, J. Polf, "Evaluation of a stochastic reconstruction algorithm for use in Compton camera imaging and beam range verification from secondary gamma emission during proton therapy," Phys. Med. Biol., vol. 57, no. 11, pp. 3537-3553, 2012.

[8] I. Perali et al., "Prompt gamma imaging of proton pencil beams at clinical dose rate," Phys. Med. Biol., vol. 59, no. 19, pp. 5849-71, 2014.

[9] C. H. Min, H. R. Lee, C. H. Kim, S. B. Lee, "Development of array-type prompt gamma measurement system for in vivo range verification in proton therapy," Med. Phys., vol. 39, no. 4, pp. 2100-07, 2012.

[10] S. W. Peterson, D. Robertson, J. Polf, "Optimizing a three-stage Compton camera for measuring prompt gamma rays emitted during proton radiotherapy," Phys. Med. Biol., vol. 55, no. 22, pp. 6841-56, 2010.

[11] F. M. F. C. Janssen et al., "Factors influencing the accuracy of beam range estimation in proton therapy using prompt gamma emission," Phys. Med. Biol., vol. 59, no. 15, pp. 4427-41, 2014.

[12] J. C. Polf et al., "Measurement and calculation of characteristic prompt gamma ray spectra emitted during proton irradiation," Phys. Med. Biol., vol. 54, no. 22, pp. N519-527, 2009.

[13] J. M. Verburg, K. Riley, T. Bortfeld, J. Seco, "Energy and time resolved detection of prompt gamma rays for proton range verification," Phys. Med. Biol., vol. 58, no. 20, pp. L37-L49, 2013.

[14] J. C. Polf, S. Peterson, G. Ciangaru, M. Gillin, S. Beddar, "Prompt gamma-ray emission from biological tissues during proton irradiation: a preliminary study," Phys. Med. Biol., vol. 54, no. 3, pp. 731-743, 2009.

[15] J. M. Verburg, J. Seco, "Proton range verification through prompt gamma-ray spectroscopy," Phys. Med. Biol., vol.59, no. 23, pp. 7089106, 2014.

[16] M. Zarifi, "Prompt gamma-ray imaging for on-line tracking of the Bragg peak in proton radiation therapy," University of Wollongong Thesis Collection, 2015.

[17] M. Zarifi, Y. Qi, S. Guatelli, B. Hutton, A. Rosenfeld, "Investigation of optimized prompt gamma detection strategy for real-time Bragg peak tracking in proton radiation therapy," Conference Record, NSS/MIC, 2015 IEEE.

[18] S. Agostinelli et al., "Geant4 - a simulation toolkit," Nucl. Instrum. Methods Phys. Res. A, vol. 506, no. 3, pp. 250-303, 2003.

[19] J. Allison et al., "Geant4 developments and applications," IEEE Trans. Nucl. Sci., vol. 53, no. 1, pp. 270-278, 2006. 